\title{
Resonant Tunneling Controlled by Laser and Constant Electric Fields
}

\author{
J.Z. KAMIŃSKI* AND E. SACZUK \\ Institute of Theoretical Physics, Faculty of Physics, University of Warsaw \\ Hoża 69, 00-681 Warszawa, Poland
}

(Received December 31, 2011)

\begin{abstract}
We develop the concept of scattering matrix and we use it to perform stable numerical calculations of resonant tunneling of electrons through a multiple potential barrier in a semiconductor heterostructure. Electrons move in two external nonperturbative electric fields: constant and oscillating in time. We apply our algorithm for different strengths and spatial configurations of the fields.
\end{abstract}

PACS: 03.65.Xp, 72.20.Dp, 73.40.Gk

\section{Introduction}

The aim of this paper is to present a numerically stable algorithm for investigations of nonrelativistic quantum processes occurring in arbitrary space-dependent scalar potential and a time- and space-dependent vector potential. Vector potential is periodic in time and describes a laser field. Such conditions are met for example in semiconductor nanostructures [1-8] (like quantum wires or wells), photoemission from a metal tip [9], carbon nanotubes $[10,11]$ or in surface physics $[12-16]$. To make our presentation as clear as possible we shall restrict ourselves to the one-space-dimensional case, although extension of this algorithm to two and three-space dimensional systems, also with magnetic field accounted for, is possible (see, e.g. [17]). We shall apply our method to investigation of the tunneling process and its dependence on relative phases of multichromatic laser pulses (multicolor processes have been considered for instance in $[16,18]$ ).

Multiple barrier, field-assisted resonant tunneling is an interesting problem because it provides an insight into the physics of nanostructure quantum systems and because it is a fundamental effect to use in a wide variety of technological applications. As concerns the latter, it is enough to mention all sorts of detectors and generators of microwave radiation based on double barrier structures with external electric field added; for more examples, see [19-24].

Here we analyze resonant tunneling through semiconductor structures in the presence of both oscillating and constant in time external electric fields. The fields are supposed to be nonperturbative. We assume that single-

* corresponding author; e-mail: jkam@fuw.edu.pl -particle states of electrons in heterostructures are well approximated by the so-called envelope function [21, 24]. Effects of sharp interfaces between different semiconductors are accounted for by boundary conditions satisfied by the envelope wave function, i.e., by the continuity of both the envelope wave function and the probability current at the interfaces (see, for example, [25-32]). Scalar potential $V(x)$ is assumed to be constant in time but it can be of any shape. The same conditions hold for space-dependent effective mass $m(x)$. Vector potential $A(x, t)$ describes a laser field and thus it is space-dependent and oscillates in time. In our approach to numerical computations, one-dimensional space is sliced into small intervals where $m(x), V(x)$ and $A(x, t)$ are space-independent. For arbitrary space-dependent functions $m(x), V(x)$ and $A(x, t)$ such a procedure is justified, provided that the widths of these intervals are sufficiently small.

We develop below a general numerical scheme which permits to evaluate transition and reflection probabilities for electrons moving in the system described above.

This paper is organized as follows. In Sect. 2 the most general solution of the Schrödinger equation is introduced. The transfer-matrix method and matching conditions are analyzed in Sect. 3, whereas reflection and transition probabilities are introduced in Sect. 4. These probabilities must sum up to 1 , which puts a very strong check for the accuracy of numerical calculations. The most important part of this paper, i.e. the concept of the scattering-matrix method, is discussed in the next section, where it is shown why the scattering-matrix algorithm has to be introduced, instead of a much simpler transfer-matrix algorithm. Numerical illustrations of the applicability of this algorithm are presented in Sects. 6 and 7 , and are followed by short conclusions. 


\section{Solution of the Schrödinger equation}

Let us start with one-dimensional Schrödinger equation of the form [21, 26],

$$
\begin{aligned}
\mathrm{i} \partial_{t} \psi(x, t) & =\left[\frac{1}{2}\left(\frac{1}{\mathrm{i}} \partial_{x}-e A(x, t)\right)\right. \\
\times & \left.\frac{1}{m(x)}\left(\frac{1}{\mathrm{i}} \partial_{x}-e A(x, t)\right)+V(x)\right] \psi(x, t) .
\end{aligned}
$$

Space-dependent mass $m(x)$, scalar potential $V(x)$ and vector potential $A(x, t)$ are spatially constant in finite intervals. Their values in any interval $\left(x_{i-1}, x_{i}\right)$ will be denoted as $m_{i}, V_{i}$ and $A_{i}(t)$. An example of such a structure is presented in Fig. 1. We require also that the func-

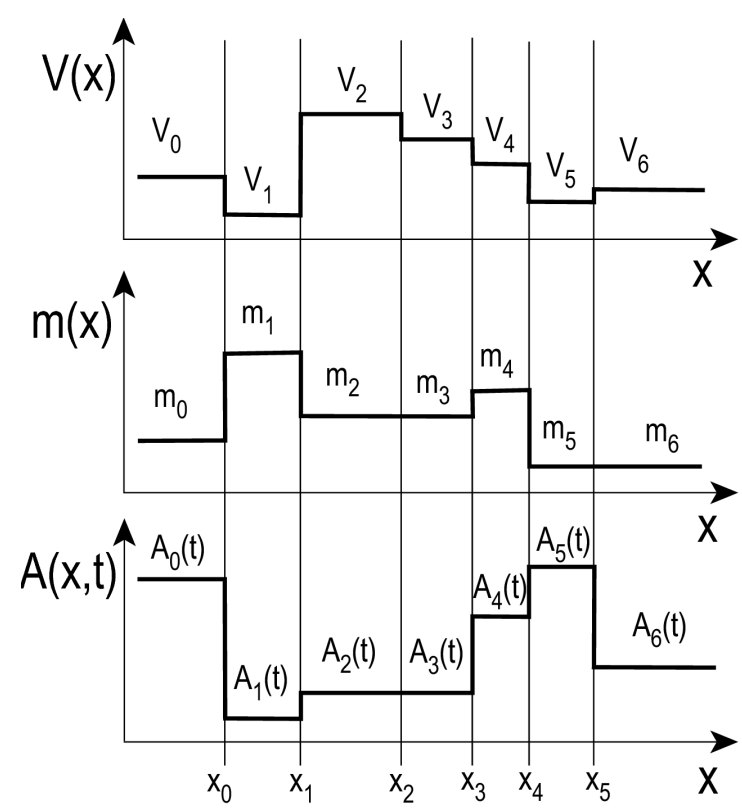

Fig. 1. Generic shapes of space-dependent superlattice potential $V(x)$, effective mass $m(x)$, and oscillating in time laser field $A(x, t)$.

tion $A(x, t)$ is periodic in time, that is

$$
A(x, t+T)=A(x, t),
$$

where $T=2 \pi / \omega$ and $\omega$ is the frequency of the oscillating in time electric field. Defining in a standard way the probability density $\rho(x, t)$,

$$
\rho(x, t)=|\psi(x, t)|^{2},
$$

and the probability current $j(x, t)$,

$$
\begin{gathered}
j(x, t)=\frac{1}{2} \psi^{*}(x, t) \frac{1}{m(x)}\left(\frac{1}{\mathrm{i}} \partial_{x}-e A(x, t)\right) \psi(x, t) \\
+\frac{1}{2} \psi(x, t) \frac{1}{m(x)}\left[\left(\frac{1}{\mathrm{i}} \partial_{x}-e A(x, t)\right) \psi(x, t)\right]^{*},
\end{gathered}
$$

we show using Eq. (1) that the conservation of probability condition is satisfied. Indeed, assuming the above definitions, we get the continuity equation,

$$
\partial_{t} \rho(x, t)+\partial_{x} j(x, t)=0 .
$$

Space dependence of mass in Eq. (1) forces one to impose non-standard continuity conditions on any solution of this equation. It is now the wave function $\psi(x, t)$ and the quantity

$$
\frac{1}{m(x)}\left(\frac{1}{\mathrm{i}} \partial_{x}-e A(x, t)\right) \psi(x, t)
$$

that have to be continuous at points of discontinuity of mass $m(x)$ and both potentials $V(x)$ and $A(x, t)$ [26-29]. Before passing to a general solution $\psi(x, t)$ of Eq. (1) in any given interval $\left(x_{i-1}, x_{i}\right)$, which we shall denote as $\psi_{i}(x, t)$, let us note that due to time periodicity of the Hamiltonian, $\psi_{i}(x, t)$ can be chosen such that the Floquet condition,

$$
\psi_{i}(x, t+T)=\mathrm{e}^{-\mathrm{i} E T} \psi_{i}(x, t),
$$

is satisfied, where $E$ is the so-called quasi-energy. A general solution $\psi_{i}(x, t)$ of Eq. (1) in any interval $\left(x_{i-1}, x_{i}\right)$ takes then the following form $[30,31]$ :

$$
\begin{aligned}
& \psi_{i}(x, t)=\sum_{M=-\infty}^{\infty} \exp (-\mathrm{i}(E+M \omega) t) \\
& \quad \times \sum_{\sigma= \pm} \sum_{N=-\infty}^{\infty} C_{i N}^{\sigma} \mathcal{B}_{M-N}\left(\sigma p_{i N}\right) \exp \left(\mathrm{i} \sigma p_{i N} x\right),
\end{aligned}
$$

where $C_{i N}^{\sigma}$ are arbitrary complex numbers to be determined and

$$
p_{i N}=\sqrt{2 m_{i}\left(E+N \omega-V_{i}-U_{i}\right)},
$$

with $U_{i}=e^{2}\left\langle A_{i}^{2}(t)\right\rangle / 2 m_{i}$ being the ponderomotive energy, where $\left\langle A_{i}^{2}(t)\right\rangle$ means the time-average of $A_{i}^{2}(t)$ over the laser-field oscillation. Components for which $p_{i N}$ are purely imaginary are called closed channels. These channels are not observed for a particle in initial or final states, but they have to be taken into account in order to satisfy the unitary condition of the time evolution. In a general case, the $\mathcal{B}_{M-N}\left(\sigma p_{i N}\right)$ functions are components of the following Fourier expansion:

$$
\exp \left(\mathrm{i} \Phi_{i N}^{\sigma}(t)\right)=\sum_{M=-\infty}^{\infty} \exp (-\mathrm{i} M \omega t) \mathcal{B}_{M-N}\left(\sigma p_{i N}\right),
$$

provided that the vector potential $A(x, t)$ is periodic in time. Functions $\Phi_{i N}^{\sigma}(t)$ are defined as follows:

$$
\begin{gathered}
\Phi_{i N}^{\sigma}(t)=\int_{0}^{t}\left[\frac{\sigma e}{m_{i}} A_{i}(t) p_{i N}\right. \\
\left.-\frac{e^{2}}{2 m_{i}}\left(A_{i}^{2}(t)-\left\langle A_{i}^{2}(t)\right\rangle\right)\right] \mathrm{d} t .
\end{gathered}
$$

It is easily seen from the above equation that the $\mathcal{B}_{M-N}\left(\sigma p_{i N}\right)$ functions depend on the form of the vector potential $A(x, t)$, that is on the laser field applied.

\section{Matching conditions and transfer matrix}

Continuity conditions discussed above and applied to a general solution (8) of the Schrödinger equation (1) lead 
to an infinite chain of equations connecting constants $C_{i N}^{\sigma}$ in the neighboring domains. These matching conditions can be written in the matrix form,

$$
B\left(i-1, x_{i-1}\right) C_{i-1}=B\left(i, x_{i-1}\right) C_{i},
$$

where $C_{i N}^{ \pm}=\left[C_{i}^{ \pm}\right]_{N}$ are the components of the columns $C_{i}^{ \pm}$. The matrices $B(i, x)$ and $C_{i}$ are defined as follows:

$$
B(i, x)=\left(\begin{array}{cc}
B^{+}(i, x) & B^{-}(i, x) \\
B^{\prime+}(i, x) & B^{\prime-}(i, x)
\end{array}\right), \quad C_{i}=\left(\begin{array}{c}
C_{i}^{+} \\
C_{i}^{-}
\end{array}\right) .
$$

The elements of $B(i, x)$ can be computed in the following way.

For an arbitrary function $A(x, t)$, periodic in time with the period $T$,

$$
A(x, t)=A(x, t+T),
$$

we have

$$
A(x, t)=\sum_{n=-\infty}^{\infty} b_{n}(x) \exp (-\mathrm{i} n \omega t),
$$

where $\omega=2 \pi / T$. In the interval $\left(x_{i-1}, x_{i}\right)$ coefficients $b_{n}(x)$ assume constant values, which we shall denote as $b_{i, n}$. Using the condition of the continuity of the wave function $\psi_{i}(x, t)$ at the point $x_{i-1}$, we compute the elements of the matrices $B^{+}$and $B^{-}$,

$$
B^{ \pm}(i, x)_{M, N}=\mathcal{B}_{M-N}\left( \pm p_{i, N}\right) \exp \left( \pm \mathrm{i} p_{i, N} x\right) .
$$

On the other hand, elements of the $B^{\prime}$ matrix can be evaluated by substituting a general solution (8) to the expression (6) and applying the continuity condition to it at $x_{i-1}$. After some algebraic manipulations we obtain the following equation:

$$
\begin{aligned}
& \frac{1}{m_{i-1}}\left(\sum_{M=-\infty}^{\infty} \exp (-\mathrm{i}(E+M \omega) t)\right. \\
& \times \sum_{\sigma= \pm 1} \sum_{N=-\infty}^{\infty} C_{i-1, N}^{\sigma} \mathcal{B}_{M-N}\left(\sigma p_{i-1, N}\right) \sigma p_{i-1, N} \\
& \times \exp \left(\mathrm{i} \sigma p_{i-1, N} x_{i-1}\right)-\sum_{M=-\infty}^{\infty} \exp (-\mathrm{i}(E+M \omega) t) \\
& \times \sum_{\sigma= \pm 1} \sum_{N, n=-\infty}^{\infty} e b_{i-1, n} C_{i-1, N}^{\sigma} \mathcal{B}_{M-N-n}\left(\sigma p_{i-1, N}\right) \\
& \left.\times \exp \left(\mathrm{i} \sigma p_{i-1, N} x_{i-1}\right)\right) \\
& =\frac{1}{m_{i}}\left(\sum_{M=-\infty}^{\infty} \exp (-\mathrm{i}(E+M \omega) t)\right. \\
& \times \sum_{\sigma= \pm 1}^{\infty} \sum_{N=-\infty}^{\infty} C_{i, N}^{\sigma} \mathcal{B}_{M-N}\left(\sigma p_{i, N}\right) \sigma p_{i, N} \\
& \times \exp \left(\mathrm{i} \sigma p_{i, N} x_{i-1}\right)-\sum_{M=-\infty}^{\infty} \exp (-\mathrm{i}(E+M \omega) t)
\end{aligned}
$$

$$
\begin{aligned}
& \times \sum_{\sigma= \pm 1} \sum_{N, n=-\infty}^{\infty} e b_{i, n} C_{i, N}^{\sigma} \mathcal{B}_{M-N-n}\left(\sigma p_{i, N}\right) \\
& \left.\times \exp \left(\mathrm{i} \sigma p_{i, N} x_{i-1}\right)\right) .
\end{aligned}
$$

Suspending the summation over $M$ on both sides of the above equation, we finally get the expression for the $B^{\prime}$ -matrices,

$$
\begin{aligned}
& B^{\prime \pm}(i, x)_{M, N}= \pm \frac{1}{m_{i}} \mathcal{B}_{M-N}\left(p_{i, N}\right) p_{i, N} \exp \left( \pm \mathrm{i} p_{i, N} x\right) \\
& -\frac{1}{m_{i}} \sum_{n=-\infty}^{\infty} e b_{i, n} \mathcal{B}_{M-N-n}\left( \pm p_{i, N}\right) \\
& \quad \times \exp \left( \pm i p_{i, N} x\right) .
\end{aligned}
$$

In this way we obtain a set of equations for vectors $C_{i}$,

$$
C_{i}=B_{i} C_{i-1},
$$

where

$$
B_{i}=\left[B\left(i, x_{i-1}\right)\right]^{-1} B\left(i-1, x_{i-1}\right) .
$$

These relations allow to connect a solution in a given domain $x_{i-1}<x<x_{i}$ with an analogous solution in any other domain $x_{j-1}<x<x_{j}$,

$$
C_{j}=B_{j} B_{j-1}, \ldots, B_{i+1} C_{i}=\mathcal{T}_{j i} C_{i},
$$

where $\mathcal{T}_{j i}$ is the so-called transfer matrix [22, 28, 30, $32,33]$.

\section{Reflection and transition probabilities}

It is clear now that on the basis of Eq. (21) we can connect solutions in the boundary domains $\left(-\infty, x_{0}\right)$ and $\left(x_{L-1}, \infty\right)$. Values of mass $m(x)$, scalar potential $V(x)$ and vector potential $A(x, t)$ in these domains will be denoted as $m_{0}, V_{0}, A_{0}(t)$ and $m_{L}, V_{L}, A_{L}(t)$, respectively. We can then write down solutions of (1) for each of these domains. These solutions represent incident $\left(\psi_{\text {inc }}\right)$, reflected $\left(\psi_{\text {ref }}\right)$ and transmitted $\left(\psi_{\text {tr }}\right)$ waves, and take the following form:

$$
\begin{aligned}
& \psi_{\text {inc }}(x, t)=\sum_{M=-\infty}^{\infty} \exp (-\mathrm{i} E t) \exp (-\mathrm{i} M \omega t) \\
& \times \mathcal{B}_{M}\left(p_{0}\right) \exp \left(\mathrm{i} p_{0} x\right) \\
& \psi_{\text {ref }}(x, t)=\sum_{N, M=-\infty}^{\infty} C_{0, N}^{-} \exp (-\mathrm{i} E t) \exp (-\mathrm{i} M \omega t) \\
& \quad \times \mathcal{B}_{M-N}\left(-p_{N}\right) \exp \left(-\mathrm{i} p_{N} x\right), \\
& \psi_{\operatorname{tr}}(x, t)=\sum_{M=-\infty}^{\infty} C_{L, N}^{+} \exp (-\mathrm{i} E t) \exp (-\mathrm{i} M \omega t) \\
& \quad \times \mathcal{B}_{M-N}\left(q_{N}\right) \exp \left(\mathrm{i} q_{N} x\right),
\end{aligned}
$$

where

$$
p_{N}=\sqrt{2 m_{0}\left(E+N \omega-V_{0}-U_{0}\right)},
$$




$$
q_{N}=\sqrt{2 m_{L}\left(E+N \omega-V_{L}-U_{L}\right)} .
$$

Constants $C_{0, N}^{-}$and $C_{L, N}^{+}$will be denoted from now on as $R_{N}$ and $T_{N}$, respectively. Using continuity conditions for functions defined above, we get the probability conservation equation for reflection and transition amplitudes, $R_{N}$ and $T_{N}$

$$
\sum_{N \geqslant N_{\text {ref }}} \frac{p_{N}}{p_{0}}\left|R_{N}\right|^{2}+\sum_{N \geqslant N_{\text {tr }}} \frac{m_{0} q_{N}}{m_{L} p_{0}}\left|T_{N}\right|^{2}=1,
$$

where summations are over such $N$ for which $p_{N}$ and $q_{N}$ are real, i.e., over the open channels. This equation permits us to interpret

$$
P_{R}(N)=\frac{p_{N}}{p_{0}}\left|R_{N}\right|^{2}
$$

and

$$
P_{T}(N)=\frac{m_{0} q_{N}}{m_{L} p_{0}}\left|T_{N}\right|^{2}
$$

as reflection and transition probabilities for a tunneling process in which absorption $(N>0)$ or emission $(N<0)$ of energy $N \omega$ by electrons occurred [30, 29]. In case of a monochromatic laser field this process can be interpreted as absorption or emission of $N$ photons from the laser field.

The unitary condition (26) can be also interpreted as the conservation of electric charge. To this end, let us define the quantities proportional to the density of electric currents,

$$
\begin{aligned}
& J_{\text {inc }}=\frac{p_{0}}{m_{0}}, \\
& J_{\text {ref }}=\sum_{N \geqslant N_{\text {ref }}} \frac{p_{N}}{m_{0}}\left|R_{N}\right|^{2}, \\
& J_{\mathrm{tr}}=\sum_{N \geqslant N_{\mathrm{tr}}} \frac{q_{N}}{m_{L}}\left|T_{N}\right|^{2} .
\end{aligned}
$$

Then Eq. (26) adopts the form of the first Kirchhoff law,

$$
J_{\text {inc }}=J_{\text {ref }}+J_{\text {tr }} \text {. }
$$

Using (21) we can calculate constants $C_{0, N}^{-}=R_{N}$ and $C_{L, N}^{+}=T_{N}$ appearing in Eqs. (22)-(24). Indeed, since

$$
C_{L}=\mathcal{T} C_{0},
$$

where transfer matrix $\mathcal{T}=\mathcal{T}_{L 0}$, and because $\mathcal{T}, C_{0}$ and $C_{L}$ adopt the following block forms:

$$
\begin{aligned}
& \mathcal{T}=\left(\begin{array}{cc}
\mathcal{T}^{++} & \mathcal{T}^{+-} \\
\mathcal{T}^{-+} & \mathcal{T}^{--}
\end{array}\right), \\
& C_{0}=\left(\begin{array}{c}
C_{0}^{+} \\
R
\end{array}\right), \quad C_{L}=\left(\begin{array}{c}
T \\
0
\end{array}\right),
\end{aligned}
$$

we arrive at

$$
T=\mathcal{T}^{++} C_{0}^{+}+\mathcal{T}^{+-} R, \quad 0=\mathcal{T}^{-+} C_{0}^{+}+\mathcal{T}^{--} R,
$$

where $R$ and $T$ denote the columns of $R_{N}$ and $T_{N}$, and $\left[C_{0}^{+}\right]_{N}=\delta_{0, N}$. Thus, after some algebraic manipulations, we have,

$$
R=-\left(\mathcal{T}^{--}\right)^{-1} \mathcal{T}^{-+} C_{0}^{+} .
$$

$$
T=\left(\mathcal{T}^{++}-\mathcal{T}^{+-}\left(\mathcal{T}^{--}\right)^{-1} \mathcal{T}^{-+}\right) C_{0}^{+},
$$

which allows us to determine the quantities $R_{N}$ and $T_{N}$ for a given transfer matrix $\mathcal{T}$. For open channels, these quantities are the amplitudes of reflection $\left(R_{N}\right)$ and transition $\left(T_{N}\right)$ probabilities, from which one can compute reflection and transition probabilities using Eqs. (27) and (28). In all our numerical illustrations, condition (26) is satisfied with an error smaller than $10^{-14}$.

\section{The scattering matrix}

We note from Eqs. (16) and (18) that each of the $B_{i}$ matrices that constitute the transfer matrix $\mathcal{T}_{j i}$ contain elements $\exp \left( \pm \mathrm{i} p_{i, N} x_{i}\right)$ that depend on the $x_{i}$ coordinates at which the discontinuities appear. For closed channels, that is when the $p_{i, N}$ momenta are purely imaginary, these numbers are real and may assume arbitrary values, very large or very small, depending again on the $x_{i}$ coordinates. Number of the $B_{i}$ matrices is equal to the number of discontinuity points, that is it depends on how we divide the space into short intervals in order to make our potential tractable by our algorithm. It may therefore turn out that in order to compute the transfer matrix $\mathcal{T}_{j i}$, we have to multiply a large number of the $B_{i}$ matrices, each containing both very small and very large numbers. It is clear that such a procedure is numerically unstable. We have to find a way to modify our method of calculations in order to compute the elements of each $B_{i}$ matrix at the same point $x=0$ independently of where the "real" $x_{i}$ is. This would eliminate "dangerous" $\exp \left( \pm \mathrm{i} p_{i, N} x_{i}\right)$ elements (turning them to 1$)$, however at the cost of appearing somewhere else. We shall see later that these "left-overs" of the shift into $x=0$ appear only as differences $x_{i+1}-x_{i}$ and therefore do not cause any harmful side-effects. We shall see now that such a modification is possible and the price we pay for it is worth the effort.

It follows from Eq. (21) that in the neighboring domains, $\left(x_{i-2}, x_{i-1}\right)$ and $\left(x_{i-1}, x_{i}\right)$, we have,

$$
C_{i}=\mathcal{T}_{i, i-1} C_{i-1} \text {. }
$$

Although the elements of the transfer matrix $\mathcal{T}_{i, i-1}$ have been computed from the continuity conditions at point $x_{i-1}$, one can compute them at any other point, for example $x=0$. To this end, let us notice what follows from the form of the solution (8). Translation of the system by a certain distance $\delta$ along the $x$-axis causes only multiplication of each member of the sum over $N$ in (8) by a constant $\exp \left(\mathrm{i} \sigma p_{i N} \delta\right)$. These constants can be included in coefficients $C_{i N}^{\sigma}$. In this way we get a new set of constants which we shall denote as $\tilde{C}_{i N}^{\sigma}$,

$$
\tilde{C}_{i N}^{\sigma}=\exp \left(\mathrm{i} \sigma p_{i N} \delta\right) C_{i N}^{\sigma} .
$$

We shall interpret these constants as coefficients in solution (8), given by the continuity conditions at point $x_{i-1}-\delta$. Equation (38) written in the matrix form becomes 
$\tilde{C}_{i}=\mathcal{P}_{i}(\delta) C_{i}$

where

$$
\mathcal{P}_{i}(\delta)=\left(\begin{array}{cc}
P_{i}^{+}(\delta) & 0 \\
0 & P_{i}^{-}(\delta)
\end{array}\right),
$$

and

$$
C_{i}=\left(\begin{array}{c}
C_{i}^{+} \\
C_{i}^{-}
\end{array}\right), \quad \tilde{C}_{i}=\left(\begin{array}{c}
\tilde{C}_{i}^{+} \\
\tilde{C}_{i}^{-}
\end{array}\right) .
$$

In the equation above $P_{i}^{\sigma}(\delta)$ is a diagonal matrix,

$$
\left[P_{i}^{\sigma}(\delta)\right]_{N N^{\prime}}=\delta_{N N^{\prime}} \exp \left(\mathrm{i} \sigma p_{i N} \delta\right),
$$

whereas $C_{i}^{ \pm}$and $\tilde{C}_{i}^{ \pm}$are the columns of the constants $C_{i N}^{ \pm}$and $\tilde{C}_{i N}^{ \pm}$, respectively, that is $\left[C_{i}^{ \pm}\right]_{N}=C_{i N}^{ \pm}$and $\left[\tilde{C}_{i}^{ \pm}\right]_{N}=C_{i N}^{ \pm}$. It follows from the form of the matrix $\mathcal{P}_{i}(\delta)$ that the following relations are satisfied:

$$
\begin{aligned}
& \mathcal{P}_{i}^{-1}(\delta)=\mathcal{P}_{i}(-\delta), \\
& \mathcal{P}_{i}\left(\delta_{1}\right) \mathcal{P}_{i}\left(\delta_{2}\right)=\mathcal{P}_{i}\left(\delta_{1}+\delta_{2}\right) .
\end{aligned}
$$

Let us notice also that translation of the system defined above modifies the transfer matrix $\mathcal{T}_{i, i-1}$. We have

$$
\begin{aligned}
& \mathcal{P}_{i}^{-1} \tilde{C}_{i}=C_{i}=\mathcal{T}_{i, i-1} C_{i-1} \\
& =\mathcal{T}_{i, i-1} \mathcal{P}_{i-1}^{-1}(\delta) \mathcal{P}_{i-1}(\delta) C_{i-1},
\end{aligned}
$$

thus

$$
\tilde{C}_{i}=\mathcal{P}_{i}(\delta) \mathcal{T}_{i, i-1} \mathcal{P}_{i-1}^{-1}(\delta) \tilde{C}_{i-1},
$$

and we can write it down as

$$
\tilde{C}_{i}=\tilde{\mathcal{T}}_{i, i-1} \tilde{C}_{i-1},
$$

where

$$
\tilde{\mathcal{T}}_{i, i-1}=\mathcal{P}_{i}(\delta) \mathcal{T}_{i, i-1} \mathcal{P}_{i-1}^{-1}(\delta) .
$$

Matrix elements denoted with the tilde symbol refer to the translated system. Using the method defined above and the relation (21), we can connect now the solution in the domain $\left(-\infty, x_{0}\right)$ with the solution in any other domain $\left(x_{i-1}, x_{i}\right)$. In this way the elements of the transfer matrix, which have been computed until now at the points of discontinuity $x_{0} \ldots x_{i-1}$, are computed now each time at the same point $x=0$. Let us illustrate this method for a special case of $i=3$ :

$$
\begin{aligned}
C_{3} & =\mathcal{T}_{3,2} \mathcal{T}_{2,1} \mathcal{T}_{1,0} C_{1}=\mathcal{P}_{3}^{-1}\left(x_{2}\right) \mathcal{T}_{3,2}^{0} \mathcal{P}_{2}\left(x_{2}\right) \mathcal{P}_{2}^{-1}\left(x_{1}\right) \\
& \times \mathcal{T}_{2,1}^{0} \mathcal{P}_{1}\left(x_{1}\right) \mathcal{P}_{1}^{-1}\left(x_{0}\right) \mathcal{T}_{1,0}^{0} \mathcal{P}_{0}\left(x_{0}\right) C_{0} \\
& =\mathcal{P}_{3}^{-1}\left(x_{2}\right) \mathcal{T}_{3,2}^{0} \mathcal{P}_{2}\left(x_{2}-x_{1}\right) \\
& \times \mathcal{T}_{2,1}^{0} \mathcal{P}_{1}\left(x_{1}-x_{0}\right) \mathcal{T}_{1,0}^{0} \mathcal{P}_{0}\left(x_{0}\right) C_{0}
\end{aligned}
$$

Equation (49) connects constants $C_{0}$ and $C_{3}$ using the matrices $\mathcal{T}_{j, j-1}^{0}$ all computed at $x=0$ independently of $j$, and diagonal matrices $\mathcal{P}_{j}\left(\delta_{j}\right)$, given by the relations (40) and (42), where $\delta_{j}=x_{j}-x_{j-1}$. Edge matrices $\mathcal{P}_{0}\left(x_{0}\right)$ and $\mathcal{P}_{3}^{-1}\left(x_{2}\right)$ in Eq. (49) can be omitted while computing the transmission and reflection probability amplitudes since their only role is to multiply the amplitudes by phase quotients which disappear while computing the probabilities. Although these matrices lead to significant modifications of the closed channels in the domains of $x<x_{0}$ and $x>x_{3}$ in this particular case, these channels do not influence the reflection and transition amplitudes. Transmission and reflection probabilities can thus be computed using a modified transfer matrix,

$$
\mathcal{T}_{3,0}^{0}=\mathcal{T}_{3,2}^{0} \mathcal{P}_{2}\left(x_{2}-x_{1}\right) \mathcal{T}_{2,1}^{0} \mathcal{P}_{1}\left(x_{1}-x_{0}\right) \mathcal{T}_{1,0}^{0} .
$$

The matrices $\mathcal{T}_{i, i-1}^{0}$ are equal to the matrices $B_{i}$ in Eq. (20) calculated however for $x_{i-1}=0$. This fact speeds up numerical calculations since now matrix $B(i, x=0)$ in Eq. (20) have to be inverted only once. Further on we shall omit the superscript 0 in $\mathcal{T}$ and the tilde over $C$ in order to simplify notation. Diagrammatic representation of the equation above is shown in Fig. 2.

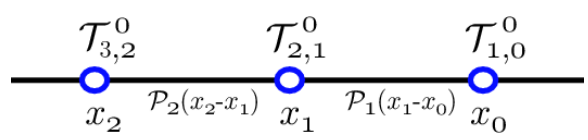

Fig. 2. Diagrammatic representation of Eq. (50). Circles represent points of discontinuity $\left\{x_{j}\right\}$ and matrices $\left\{\mathcal{T}_{j+1, j}^{0}\right\}$, whereas lines represent "free-propagators" $\left\{\mathcal{P}_{j+1}\left(x_{j+1}-x_{j}\right)\right\}$. It is important to notice that all matrices $\left\{\mathcal{T}_{j+1, j}^{0}\right\}$ are calculated at $x=0$, which prevents the development of numerical overflows.

The method presented above is still numerically unstable. The reason for this instability lies in the existence of large numerical values of elements of the $\mathcal{P}_{i}^{-}(\delta)$ matrix for imaginary momenta $p_{i N}$. In other words, for

$$
\begin{aligned}
C_{i} & =\left(\begin{array}{c}
C_{i}^{+} \\
C_{i}^{-}
\end{array}\right)=\mathcal{T}_{i, i-1} C_{i-1} \\
& =\left(\begin{array}{cc}
\mathcal{T}_{i, i-1}^{++} & \mathcal{T}_{i, i-1}^{+-} \\
\mathcal{T}_{i, i-1}^{-+} & \mathcal{T}_{i, i-1}^{--}
\end{array}\right)\left(\begin{array}{c}
C_{i-1}^{+} \\
C_{i-1}^{-}
\end{array}\right),
\end{aligned}
$$

the source of numerical instabilities are matrix elements $\mathcal{T}_{i, i-1}^{--}$that contain large numbers. There is however a chance for improving the stability, if only its reverse will be used, $\left(\mathcal{T}_{i, i-1}^{--}\right)^{-1}$. It appears that it is possible provided that in our numerical algorithm only the so-called scattering matrix will be applied. For this reason we will show below how to compute the scattering matrix, $\mathcal{S}_{j, i}$, using only elements of the transfer matrix, $\mathcal{T}_{j, i}$. For the transfer matrix $\mathcal{T}_{j, i}$ we have,

$$
\mathcal{T}_{j, i} C_{i}=C_{j}=\left(\begin{array}{c}
C_{j}^{+} \\
C_{j}^{-}
\end{array}\right)=\left(\begin{array}{cc}
\mathcal{T}_{j, i}^{++} & \mathcal{T}_{j, i}^{+-} \\
\mathcal{T}_{j, i}^{-}+ & \mathcal{T}_{j, i}^{--}
\end{array}\right)\left(\begin{array}{c}
C_{i}^{+} \\
C_{i}^{-}
\end{array}\right) .
$$

Thus,

$$
\begin{aligned}
& C_{j}^{+}=\mathcal{T}_{j, i}^{++} C_{i}^{+}+\mathcal{T}_{j, i}^{+-} C_{i}^{-}, \\
& C_{j}^{-}=\mathcal{T}_{j, i}^{-+} C_{i}^{+}+\mathcal{T}_{j, i}^{--} C_{i}^{-} .
\end{aligned}
$$

On the basis of (53) we now want to compute the elements of the $\mathcal{S}_{j, i}$ matrix. This matrix is supposed to connect the coefficients $C_{i}^{ \pm}$and $C_{j}^{ \pm}$in the following way (for the graphical illustration, see Fig. 3), 


$$
\left(\begin{array}{c}
C_{i}^{-} \\
C_{j}^{+}
\end{array}\right)=\left(\begin{array}{cc}
\mathcal{S}_{j, i}^{++} & \mathcal{S}_{j, i}^{+-} \\
\mathcal{S}_{j, i}^{-+} & \mathcal{S}_{j, i}^{--}
\end{array}\right)\left(\begin{array}{c}
C_{i}^{+} \\
C_{j}^{-}
\end{array}\right)
$$

Using the set of linear Eqs. (53), we easily compute the coefficients $C_{i}^{-}$and $C_{j}^{+}$on the left-hand side of Eq. (54), as functions of the coefficients $C_{j}^{-}$and $C_{i}^{+}$. We get then the following relations:

$$
\begin{aligned}
& C_{i}^{-}=\left(\mathcal{T}_{j, i}^{--}\right)^{-1}\left(C_{j}^{-}-\mathcal{T}_{j, i}^{-+} C_{i}^{+}\right), \\
& C_{j}^{+}=\left(\mathcal{T}_{j, i}^{++}-\mathcal{T}_{j, i}^{+-}\left(\mathcal{T}_{j, i}^{--}\right)^{-1} \mathcal{T}_{j, i}^{-+}\right) C_{i}^{+} \\
& \quad+\mathcal{T}_{j, i}^{+-}\left(\mathcal{T}_{j, i}^{--}\right)^{-1} C_{j}^{-} .
\end{aligned}
$$

Finally we compute the elements of the matrix $\mathcal{S}_{j, i}$,

$$
\begin{aligned}
\mathcal{S}_{j, i}^{++} & =-\left(\mathcal{T}_{j, i}^{--}\right)^{-1} \mathcal{T}_{j, i}^{-+}, \\
\mathcal{S}_{j, i}^{+-} & =\left(\mathcal{T}_{j, i}^{--}\right)^{-1}, \\
\mathcal{S}_{j, i}^{-+} & =\left(\mathcal{T}_{j, i}^{++}-\mathcal{T}_{j, i}^{+-}\left(\mathcal{T}_{j, i}^{--}\right)^{-1} \mathcal{T}_{j, i}^{-+}\right), \\
\mathcal{S}_{j, i}^{--} & =\mathcal{T}_{j, i}^{+-}\left(\mathcal{T}_{j, i}^{--}\right)^{-1} .
\end{aligned}
$$

As expected, the matrix $\mathcal{S}_{j, i}$ contains only numerically stable elements $\left(\mathcal{T}_{j, i}^{--}\right)^{-1}$.

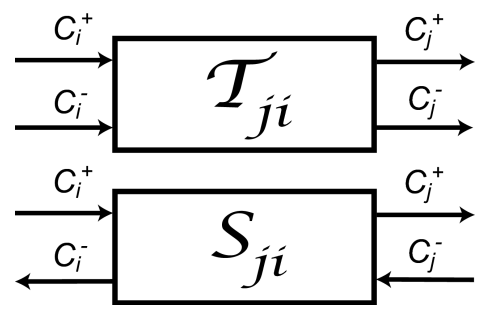

Fig. 3. Schematic representation of the idea of the transfer matrix and the scattering matrix. For the transfer matrix the incoming channels are $C_{i}^{+}$and $C_{i}^{-}$, and the outgoing channels are $C_{j}^{+}$and $C_{j}^{-}$. For the scattering matrix $C_{i}^{+}$and $C_{j}^{-}$are the incoming channels with the remaining two considered as the outgoing ones.

It follows from Eq. (21) that the transfer matrix $\mathcal{T}_{j, i}$ can be written as the product of two transfer matrices, $\mathcal{T}_{j, k}$ and $\mathcal{T}_{k, i}(i<k<j)$,

$$
\mathcal{T}_{j, i}=\mathcal{T}_{j, k} \mathcal{T}_{k, i}
$$

where matrices $\mathcal{T}_{j, k}$ and $\mathcal{T}_{k, i}$ are defined as follows:

$$
\begin{aligned}
C_{k} & =\mathcal{T}_{k, i} C_{i}, \\
C_{j} & =\mathcal{T}_{j, k} C_{k} .
\end{aligned}
$$

Applying the method presented above, for each of the transfer matrices $\mathcal{T}_{j, k}$ and $\mathcal{T}_{k, i}$ we can construct a scattering matrix, $\mathcal{S}_{j, k}$ and $\mathcal{S}_{k, i}$, respectively. Elements of the scattering matrix $\mathcal{S}_{j, i}$ can be computed using only elements of the matrices $\mathcal{S}_{j, k}$ and $\mathcal{S}_{k, i}$. Using the notation above, we obtain the following expressions for the elements of the $\mathcal{S}_{j, i}$ matrix:

$$
\mathcal{S}_{j, i}^{++}=\mathcal{S}_{k, i}^{++}+\mathcal{S}_{k, i}^{+-}\left(1-\mathcal{S}_{j, k}^{++} \mathcal{S}_{k, i}^{--}\right)^{-1} \mathcal{S}_{j, k}^{++} \mathcal{S}_{k, i}^{-+},
$$

$$
\begin{aligned}
& \mathcal{S}_{j, i}^{+-}=\mathcal{S}_{k, i}^{+-}\left(1-\mathcal{S}_{j, k}^{++} \mathcal{S}_{k, i}^{--}\right)^{-1} \mathcal{S}_{j, k}^{+-}, \\
& \mathcal{S}_{j, i}^{-+}=\mathcal{S}_{j, k}^{-+}\left(1-\mathcal{S}_{j, k}^{++} \mathcal{S}_{k, i}^{--}\right)^{-1} \mathcal{S}_{k, i}^{-+}, \\
& \mathcal{S}_{j, i}^{--}=\mathcal{S}_{j, k}^{--}+\mathcal{S}_{j, k}^{-+} \mathcal{S}_{k, i}^{--}\left(1-\mathcal{S}_{j, k}^{++} \mathcal{S}_{k, i}^{--}\right)^{-1} \mathcal{S}_{j, k}^{+-} .
\end{aligned}
$$

It is clear from the above that the $\mathcal{S}_{j, i}$ matrix is not merely a product of two matrices $\mathcal{S}_{j, k}$ and $\mathcal{S}_{k, i}$, but rather a complicated nonlinear composition of them. It is important however to note that despite its evident complexity, such a construction of the scattering matrix is numerically stable, as opposed to the transfer matrix method which fails if a system with a large number of discontinuity points $x_{i}$ is considered. Stability of such an algorithm has been proven in our numerical investigations by checking that the condition (26) is satisfied with an error smaller than $10^{-14}$. Such an accuracy can never be achieved for systems with a large number of discontinuity points if the transfer matrix is applied.

\section{Resonant tunneling}

We shall consider now the tunneling phenomenon through a semiconductor heterostructure presented in Fig. 4. In the beginning, let us assume that electrons interact only with a constant electric field. Hence, the time-independent potential is of the form $V(x)+F x$, in which $V(x)$ represents the semiconductor heterostructure potential (Fig. 4 ) and $F$ is the electric-field strength. The plot of this potential is presented in Fig. 5, where $a=40 \AA, b=20 \AA$, and $F=-0.23 \times 10^{-4}$ (in atomic units). Applying the theory developed above, we calculated reflection and transmission probabilities (see Fig. 6) discretizing the potential with 15,141 , and 281 points, as indicated in one of the frames. We observe that, in order to get convergence, one has to introduce at least one hundred discontinuity points. There is no noticeable difference between the results obtained for 141 and 281 such points.

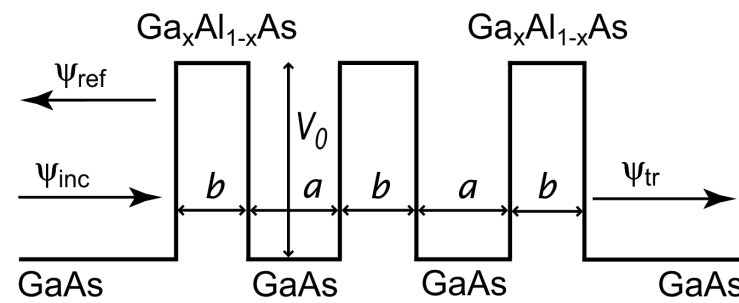

Fig. 4. Tunneling process considered in this paper. Parameters for the triple barrier are: $V_{0}=237 \mathrm{meV}$, and the effective masses $m_{\mathrm{GaAs}}=0.0667 m_{\mathrm{e}}$ and $m_{\mathrm{Ga}_{x} \mathrm{Al}_{1-x} \mathrm{As}}=0.0918 m_{\mathrm{e}}$, where $m_{\mathrm{e}}$ is the electron rest mass. The widths of the barriers $b$ and wells $a$ can change.

Next, let us analyze transmission of electrons through the triple barrier of Fig. 4 with $a=70 \AA$ and $b=20 \AA$ and with the 221 discretization points. Now we apply a constant electric field and the monochromatic laser field 


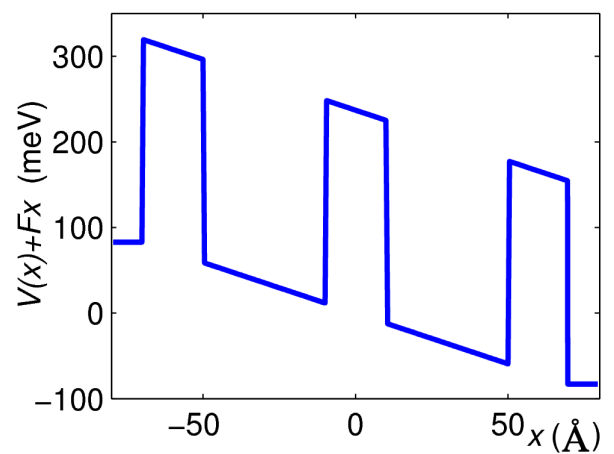

Fig. 5. Plot of the potential $V(x)+F x$ for $a=40 \AA$, $b=20 \AA$, and $F=-0.23 \times 10^{-4}$ (in atomic units). Other parameters are the same as in Fig. 4 .

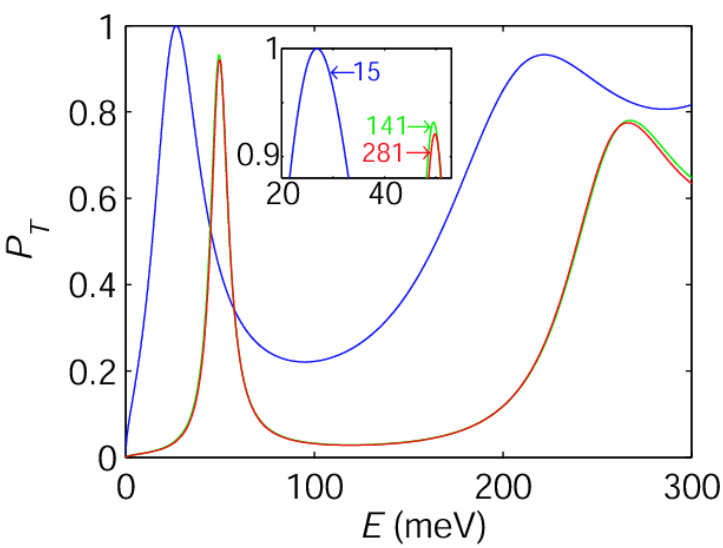

Fig. 6. Transmission probabilities for the potential shown in Fig. 5. Numbers in the inset frame (representing enlarged part of the main frame) indicate the number of equally spaced discontinuity points introduced in our numerical algorithm. We see that 15 points do not give correct results and that the convergence is reached with more than 100 points.

of frequency $\omega=70 \mathrm{meV}$ and intensity such that its ponderomotive energy divided by the laser photon energy equals $10^{-4}$. Without external fields, the resonant energies are grouped in doublets in which the lower-energy resonance corresponds to the antisymmetric resonance state, and the upper-energy resonance to the symmetric one. With the laser field switched on, this structure does not change very much provided that the frequency is off-resonance with respect to the already existing resonance states of the triple barrier, and the intensity is not too large, as it is presented in Figs. 7 and 8. The pattern changes significantly if a constant electric field is applied. We observe that with an increasing strength of the electric field the low-energy transmission resonances from a given doublet gradually disappear and we are left with a single transmission resonance, which for even stronger electric fields also dies out. This means that by proper tuning the strength of a constant electric field one can selectively transmit electrons of some particular energies.

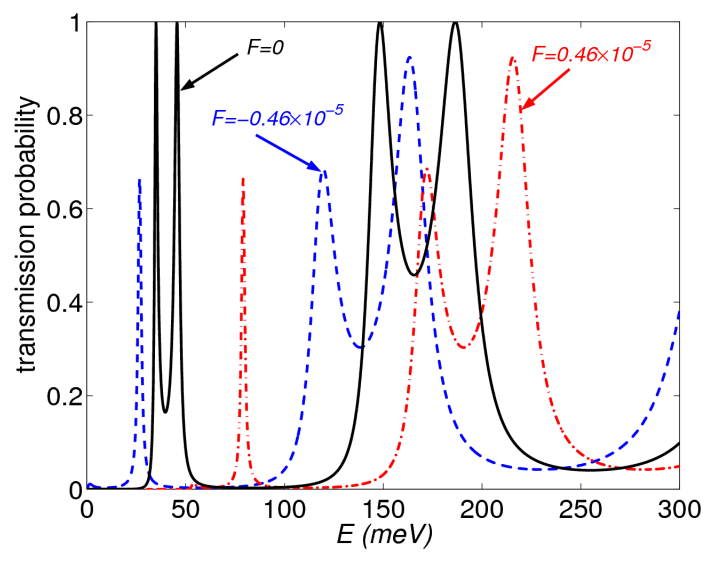

Fig. 7. Transmission probabilities for semiconductor triple barrier with $a=70 \AA, b=20 \AA$. Intensity of the laser field is such that the ratio of ponderomotive energy to photon energy is $U_{\mathrm{p}} / \omega=10^{-4}$ with $\omega=70 \mathrm{meV}$ and we have three electric-field strengths (in atomic units), as indicated in the figure. As expected, transition probability distributions for $\pm F$ [blue (dash-dash) and red (dash-dot) lines] are shifted in energy by $|F|(3 b+2 a)$. Computations were performed for 221 discretization points.

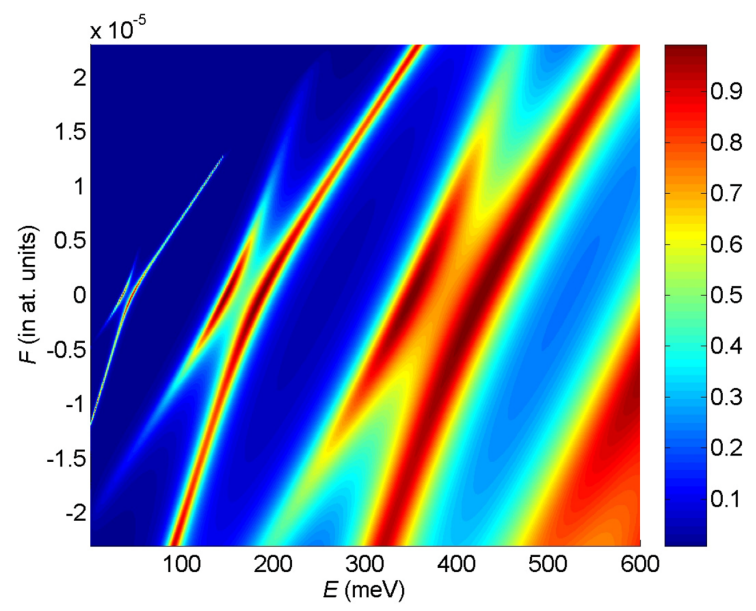

Fig. 8. Color map of the total transmission probability in the plane of the incident electron energy $E$ and the electric-field strength $F$. As expected, for the vanishing electric field, resonances in the considered in Fig. 7 triple barrier structure show up in doublets. However, for sufficiently strong electric field the lower-energy resonance from a doublet disappears.

\section{Phase control of tunneling}

Special features of barrier problems stem from the interaction of waves reflected from or transmitted through potential jumps. When the interference of reflected waves is in phase, transmission becomes minimal. But when the interference of reflected waves is out of phase (i.e., it is destructive) the incident wave resonantly penetrates either by tunneling through or passing above the barrier 
structure. If the process occurs in a monochromatic laser field the destructive or constructive interferences between reflected and transmitted waves are present also for different Fourier components of the electron wave function. This leads for example to opening or closing gaps in the band structure [1-5, 31] or formation of multiple-plateau structures in the high-order harmonic spectrum $[34,35]$. It gets even more complicated if multichromatic laser fields or short laser pulses are applied. In the first case the interference discussed above can be controlled by relative phases of harmonics present in the multichromatic fields, whereas in the second case the resonance transmission can be modified by the carrier-envelope phase.

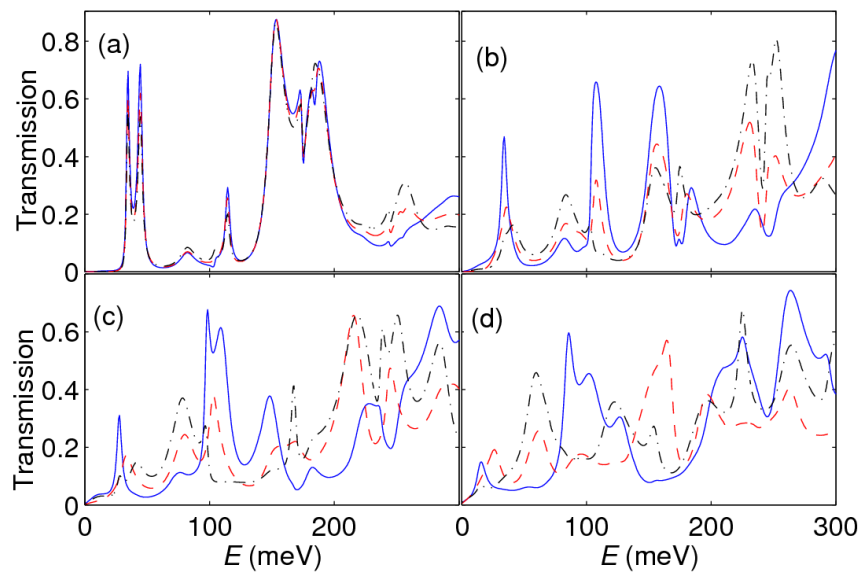

Fig. 9. Total transmission probabilities for the triple barrier structure presented in Fig. 4 with $a=70 \AA$ and $b=20 \AA$, and for the bichromatic field [Eq. (60)] with $\omega=70 \mathrm{meV}$. The continuous (blue) line is for $\varphi=$ 0 , dash-dash (red) line for $\varphi=\pi / 2$, whereas dash-dot (black) line for $\varphi=\pi$. Frames correspond to different laser field intensities characterized by the dimensionless parameter $\xi=|e| E_{0}(x) /\left(2 \sqrt{\hbar m_{\mathrm{e}} \omega^{3}}\right)$ ( $m_{\mathrm{e}}$ is the electron rest mass and $E_{0}(x)$ is considered to be constant in the whole space): (a) $\xi=0.1$, (b) $\xi=0.5$, (c) $\xi=1$, and (d) $\xi=2$.

As an example let us consider a bichromatic laser field. Let the electric field be of the form

$$
E(x, t)=E_{0}(x)[\sin (\omega t)-\sin (2 \omega t+\varphi)],
$$

where $E_{0}(x)$ is in general a space-dependent amplitude of the laser field. In Figs. 9 and 10 the laser-modified total transmission probabilities through a triple-barrier structure are presented for three different relative phases $\varphi$. Figure 9 corresponds to the situation in which the laser field acts in the whole space, whereas Fig. 10 illustrates the action of the laser field concentrated within the structure, hence incident, reflected and transmitted electrons are free. Apart from a significant dependence of these probabilities on the relative phase we observe also that in the second case and for higher intensities considered the transmission probabilities are smaller. It is because the electrons have to traverse an extra "potential barrier" created by the ponderomotive energy of a laser field in

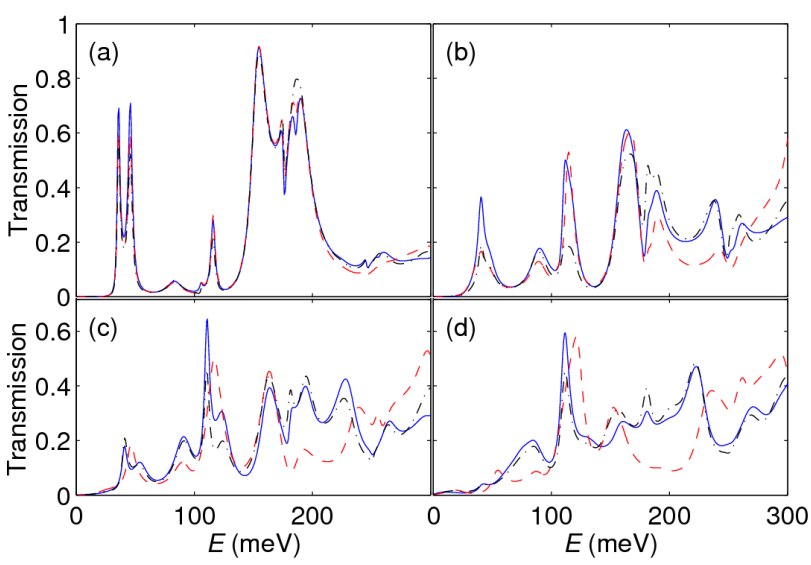

Fig. 10. The same as in Fig. 9, but with space-dependent intensity of a laser field. Now, $E_{0}(x)=E_{0}$ within the triple barrier structure, $E_{0}(x)=E_{0} / 2$ within the edge barriers and 0 outside. The electric field strength $E_{0}$ is determined by a dimensionless parameter $\xi=|e| E_{0} /\left(2 \sqrt{\hbar m_{\mathrm{e}} \omega^{3}}\right)$, with the same numerical values as in Fig. 9 .

order to transmit through barriers. This finding opens up a possibility to create tunneling barrier structures by laser fields modulated in space. This can be investigated numerically by applying the algorithm developed in this paper.

As a second example of the phase control let us consider transmission of electrons through a triple barrier structure in the presence of both a constant electric field and a train of very short laser pulses. It is well-known from atomic and molecular physics that the ionization process can be significantly modified by the so-called carrier-envelope phase if a single pulse contains only few oscillations. In order to investigate this phenomenon for electron transmission let us assume that the train of pulses is built from a single pulse (defined for times $\left.0 \leqslant t \leqslant T_{\mathrm{p}}\right)$ of the form

$$
E(x, t)=E_{0}(x) f(t) \sin (\omega t+\varphi)-\Delta_{L},
$$

where the envelope function $f(t)$ equals

$$
f(t)=\exp \left(-\left(\frac{t-T_{\mathrm{p}} / 2}{\sigma_{\mathrm{p}}}\right)^{2}\right) \sin ^{2}\left(\frac{\pi t}{T_{\mathrm{p}}}\right),
$$

and the constant in time $\Delta_{L}$ is chosen such that

$$
\int_{0}^{T_{\mathrm{p}}} E(x, t) \mathrm{d} t=0 .
$$

The carrier-envelope phase $\varphi$ can change from 0 to $2 \pi$.

In Fig. 11 we present transmission probabilities for the electrons impinging from the right on the triple barrier structure shown in Fig. 5 (however, with different values for $a$ and $F$ ). Without the action of the laser pulse the transmission is forbidden for energies smaller than approximately $100 \mathrm{meV}$, whereas for larger energies electrons can tunnel resonantly. The presence of the laser field modifies these conditions and they get similar to 


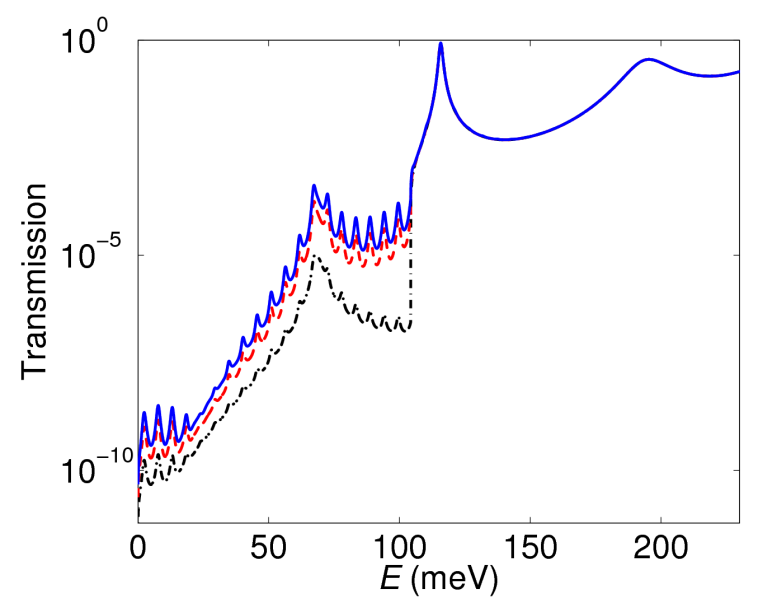

Fig. 11. Total transmission probabilities for the triple barrier structure presented in Fig. 4 with $a=70 \AA$, $b=20 \AA$, and $V_{0}=237 \mathrm{meV}$. The train of laser pulses [Eqs. (61) and (62)] with $\omega=70 \mathrm{meV}, T_{\mathrm{p}}=26 \pi / \omega$ and $\sigma_{\mathrm{p}}=T_{\mathrm{p}} / 140$ (one-cycle pulse) is defined by the space-dependent electric field such that $E_{0}(x)=E_{0}$ within the triple barrier structure, $E_{0}(x)=E_{0} / 2$ within the edge barriers and 0 outside. The laser field intensity is characterized by the dimensionless parameter $\xi=|e| E_{0} /\left(2 \sqrt{\hbar m_{\mathrm{e}} \omega^{3}}\right)$ ( $m_{\mathrm{e}}$ is the electron rest mass), whereas the constant electric field strength $F$ is determined by the parameter $\eta=|e| F(3 b+2 a) / V_{0}$. In this illustration $\xi=0.2$ and $\eta=0.1$. The continuous (blue) line is for $\varphi=\pi / 2$, dash-dash (red) line for $\varphi=\pi / 4$, whereas dash-dot (black) line for $\varphi=0$.

those met in the photoemission from solid surfaces or the ionization of atoms or molecules. For the latter it is well-known that the carrier-envelope phase substantially modifies ionization probabilities [36, 37]. The results presented here also confirm this effect for the tunneling phenomena. Transmission probabilities (hence, also photocurrents emitted from the surface) can then be changed by the carrier-envelope phase even by two orders of magnitude.

\section{Conclusions}

As mentioned above, our algorithm is convergent provided that a sufficient number of discretization points is introduced. For systems considered here, this number should not be smaller than 100 . If the laser field is very weak, this does not create significant numerical problems, except that calculations become longer. However, when the laser field is sufficiently intense, the algorithm based on the transfer matrix is unstable. This instability is due to the existence of closed channels, which introduce into numerical calculations very small and very large numbers at the same time. Augmenting precisions significantly slows down the calculation and does not diminish the problem. We have found that it is possible to make this algorithm numerically stable by just applying nonlinear matrix transformations, without introducing higher precisions.
Illustrations presented in this paper show that tunneling of electrons through multibarrier semiconductor structures can be changed significantly by applied nonperturbative electric fields: oscillating in time or constant. The efficiency of the algorithm presented in this contribution opens up the possibility of investigating surface phenomena (like photoemission or high-order harmonic generation) in the presence of more realistic laser pulses that gradually decrease within solids and extend on a mesoscopic scale in vacuum. These problems have been partly investigated for instance in [38, 39]. Further investigations in particular in the context of photoemission form a metal tip [9] and threshold effects in multiphoton processes $[40,41]$ are planned to be carried out.

\section{References}

[1] N. Tzoar, J.I. Gersten, Phys. Rev. B 12, 1132 (1975).

[2] M.R. Belić, Solid State Commun. 62, 817 (1987).

[3] F.H.M. Faisal, R. Genieser, Phys. Lett. A 141, 297 (1989).

[4] J.Z. Kamiński, Acta Phys. Pol. A 83, 495 (1993).

[5] D.R. Mašović, M.R. Belić, J.I. Gersten, Phys. Lett. A 373, 3289 (2009).

[6] R.P. Lungu, Phys. Scr. 75, 206 (2007).

[7] R. Lefebvre, Int. J. Quant. Chem. 106, 2848 (2006).

[8] R. Lefebvre, O. Atabek, J. Phys. B 38, 2133 (2005).

[9] M. Krüger, M. Schenk, P. Hommelhoff, Nature 475, 78 (2011).

[10] H. Khosravi, N. Daneshfar, A. Bahari, Opt. Lett. 34, 1723 (2009).

[11] H. Hsu, L.E. Reichl, Phys. Rev. B 74, 115406 (2006); ibid., 72, 155413 (2005).

[12] M. Faraggi, I. Aldazabal, M.S. Gravielle, A. Arnau, V.M. Silkin, J. Opt. Soc. Am. B 26, 2331 (2009).

[13] G. Saathoff, L. Miaja-Avila, M. Aeschlimann, M.M. Murname, H.C. Kapteyn, Phys. Rev. A 77, 022903 (2008).

[14] J.C. Baggesen, L.B. Madsen, Phys. Rev. A 78, 032903 (2008).

[15] M.N. Faraggi, M.S. Gravielle, D.M. Mitnik, Phys. Rev. A 76, 012903 (2007).

[16] P. Dombi, F. Krausz, G. Farkas, J. Mod. Opt. 53, 163 (2006).

[17] F.H.M. Faisal, J.Z. Kamiński, E. Saczuk, Phys. Rev. A 72, 023412 (2005); Laser Phys. 16, 272 (2006).

[18] V.A. Astapenko, Quantum Electron. 36, 1131 (2006).

[19] M. Razavy, Quantum Theory of Tunneling, World Sci., Singapore 2003.

[20] K.F. Brennan, A.S. Brown, Theory of Modern Electronic Semiconductor Devices, Wiley, New York 2002.

[21] J.H. Davies, The Physics of Low-dimensional Semiconductors. An Introduction, Cambridge University Press, Cambridge 1998.

[22] R. Tsu, L. Esaki, Appl. Phys. Lett. 22, 562 (1973).

[23] D.K. Roy, Quantum Mechanical Tunneling and Its Applications, World Sci., Singapore 1986. 
[24] C. Weisbuch, B. Vinter, Quantum Semiconductor Structures. Fundamentals and Applications, Academic Press, Boston 1991.

[25] W. Schäfer, M. Wegener, Semiconductor Optics and Transport Phenomena, Springer, Berlin 2002.

[26] J.-M. Lévy-Leblond, Eur. J. Phys. 13, 215 (1992).

[27] J.Z. Kamiński, F. Ehlotzky, J. Phys. B 32, 3193 (1999).

[28] N. Moiseyev, R. Lefebvre, Phys. Rev. A 64, 052711 (2001).

[29] E. Saczuk, J.Z. Kamiński, Phys. Status Solidi B 240, 603 (2003).

[30] J.Z. Kamiński, Z. Phys. D 16, 153 (1990).

[31] F.H.M. Faisal, J.Z. Kamiński, Phys. Rev. A 56, 748 (1997).

[32] V. Leon, R. Lefebvre, O. Atabek, Phys. Rev. A 64, 052105 (2001).

[33] R. Lefebvre, N. Moiseyev, Phys. Rev. A 69, 062105 (2004).

[34] F.H.M. Faisal, J.Z. Kamiński, Phys. Rev. A 54, R1769 (1996); ibid., 58, R19 (1998).
[35] F.H.M. Faisal, J.Z. Kamiński, S.S.M. Soliman, Laser Phys. 8, 129 (1998).

[36] G.G. Paulus, F. Grasbon, H. Walther, P. Villoresi, M. Nisoli, S. Stagira, E. Priori, S. De Silvestri, Nature 414, 182 (2001); G.G. Paulus, F. Lindner, H. Walther, A. Baltuška, E. Goulielmakis, M. Lezius, F. Krausz, Phys. Rev. Lett. 91, 253004 (2003).

[37] D.B. Milošević, G.G. Paulus, D. Bauer, W. Becker, J. Phys. B 39, R203 (2006).

[38] J.Z. Kamiński, E. Saczuk, Phys. Lett. A 375, 251 (2011).

[39] J.Z. Kamiński, J. Opt. Soc. Am. B 28, 949 (2011).

[40] K. Krajewska, I.I. Fabrikant, A.F. Starace, Phys. Rev. A 74, 053407 (2006); Phys. Rev. A 78, 023407 (2008); Laser Phys. 17, 368 (2007).

[41] L.A. Lompre, G. Mainfray, C. Manus, J. Thebault, Gy. Farkas, Z. Horvath, Appl. Phys. Lett. 33, 124 (1978). 